\title{
Energy Efficient MIMO SWIPT by Hybrid Analog-Digital Beamforming
}

\author{
Ang Li and Christos Masouros \\ Dept. of Electronic and Electrical Eng., University College London, London, U.K. \\ Email: \{ang.li.14, c.masouros\}@ucl.ac.uk
}

\begin{abstract}
In this paper, we investigate the simultaneous wireless information and power transfer (SWIPT) for MIMO systems with limited RF chains at the base station. We focus on the scenario where there is one information decoder with a targeted SINR and several separate energy receivers with energy harvesting thresholds. Based on the observation that the fully-digital beamformer consists of only the information beamformer, we propose an iterative hybrid analog-digital beamforming scheme, where we design the analog beamformer by minimizing the difference between the fully-digital beamformer and the hybrid beamformer, and the optimal solution can be obtained via a geometrical interpretation. Numerical results show that the proposed scheme can achieve a close-to-optimal performance with significant gains in the total power consumption over fully-digital SWIPT.
\end{abstract}

Index Terms-MIMO, SWIPT, hybrid beamforming, iterative design.

\section{INTRODUCTION}

Simultaneously wireless information and power transfer (SWIPT) [1] have been proposed to address the increasing energy demands of wireless components.. Three different types of energy receivers, i.e. separate, time-switching, and power splitting, are considered for SWIPT in [2], and SWIPT techniques with multiple-input multiple-output (MIMO) have drawn increasing research attention recently [3]-[6]. Zeroforcing beamforming for MIMO SWIPT is considered in [6] and it is shown that the energy harvested by the energy harvesting $(\mathrm{EH})$ receivers can be increased at the cost of a signal-to-interference-plus-noise (SINR) loss of the information decoders (IDs). Robust beamforming for MIMO SWIPT with imperfect channel state information (CSI) is further proposed in [7][8]. A data-aided transmit beamforming scheme by exploiting the constructive interference for MIMO SWIPT is proposed in [9] to offer additional performance gains. SWIPT techniques for MIMO interference channels have been proposed in [10][11], and the combinations with secure tranmission for MIMO SWIPT are investigated in [12][13].

The above studies on MIMO SWIPT all assume a fullydigital beamformer that requires a dedicated radio frequency (RF) chain for each antenna element, which leads to a low power efficiency at the BS. Even with moderate number of antennas, the power consumption of RF chains is dominant. To address this and achieve energy-efficient transmission, hybrid analog-digital beamforming schemes have been proposed by allowing a reduced number of RF chains and dividing the beamforming in the analog domain and digital domain [14]-
[18]. In the analog domain, phase-shifters are applied to provide high-dimensional phase-only controls, while a lowdimensional fully-digital beamformer is applied in the digital domain to manage interference. It has been shown that the fully-connected hybrid structures can achieve a similar performance to fully-digital beamforming schemes for both single-user case [14] and multi-user case [19], with greatly reduced total power consumption. Nevertheless, the fullyconnected hybrid structures where each antenna element is connected to all RF chains require a large number of phase shifters and switches, which is complicated and will incur significant insertion losses in practice [20][21]. To alleviate these losses and further simplify the hybrid design, partiallyconnected structures where each antenna element is only connected to one RF chain are introduced [22], at the cost of a performance loss [23]. While the hybrid beamforming is initially proposed for massive MIMO systems, the reduction in the hardware complexity and power consumption directly applies to small-scale MIMO systems. Indeed, small access points for the future internet of things (IoTs) or small BSs (for example femtocells or picocells that are widely deployed for heterogeneous networks) usually have limited power supply, which can benefit from the hybrid structures. Interestingly, such power-efficient approaches by hybrid beamforming have yet to be explored for SWIPT.

Therefore in this paper, we investigate the MIMO SWIPT systems with limited RF chains at the BS, where the hybrid analog-digital beamforming scheme is applied. We consider the scenario where there is one ID and several separate $\mathrm{EH}$ receivers, and focus on the transmit power minimization subject to the received SINR requirement of the ID and the harvested energy requirement by each $\mathrm{EH}$ receiver. Based on the observation that the optimal fully-digital beamformer consists of only the information beamformer, we propose an iterative design where we optimize the analog beamformer and the digital beamformer alternately. By minimizing the distance between the fully-digital beamformer and the hybrid beamformer, the optimal analog beamformer can be obtained within each iteration via a geometrical interpretation. We further extend the proposed analog beamformer design to partially-connected structures, where a simplified algorithm can be obtained. Numerical results show that the proposed schemes achieve a very close-to-optimal performance for fully-connected structures, and significant gains can be observed compared to existing hybrid schemes. It is also ob- 
served that the hybrid structures consume much reduced power for the BS compared to the fully-digital case.

Notations: $a$, a, and $\mathbf{A}$ denote scalar, vector and matrix, respectively. $(\cdot)^{T},(\cdot)^{H}, \operatorname{rank}\{\cdot\}$, and $\operatorname{tr}\{\cdot\}$ denote transposition, conjugate transposition, rank and trace of a matrix respectively. $\|\cdot\|$ denotes the Frobenius norm, $\mathcal{C}^{n \times n}$ represents an $n \times n$ matrix in the complex set, and $\operatorname{diag}(\cdot)$ denotes the conversion of a vector into a diagonal matrix with the values on its main diagonal. $\mathbf{R} \succeq 0$ means that $\mathbf{R}$ is a Hermitian positive semidefinite matrix.

\section{System Model And Fully-Digital SWIPT}

We consider a downlink MU-MISO system, where a BS with $N_{t}$ antennas and $N_{R F}^{t}$ RF chains serves one ID and $K$ EH receivers. Following [14][19], we assume the number of $\mathrm{RF}$ chains is equal to the total number of users, i.e. $N_{R F}^{t}=(K+1)$. Perfect CSI is assumed throughout this paper. A spatially uncorrelated Rayleigh flat-fading channel is assumed, where we denote $\mathbf{h}_{I}, \mathbf{h}_{E}^{k} \in \mathcal{C}^{1 \times N_{t}}, k \in \mathcal{K}$ as the channel vector from the $\mathrm{BS}$ to the ID and $k$-th $\mathrm{EH}$ receiver respectively, where $\mathcal{K}=\{1, \ldots, K\} . \mathbf{h}_{I}$ and $\mathbf{h}_{E}^{k}$ are then modelled as [24]

$$
\mathbf{h}_{I}=\sqrt{\alpha_{0} D_{I}^{-\beta} C_{I}} \cdot \mathbf{g}_{I}, \mathbf{h}_{E}^{k}=\sqrt{\alpha_{0}\left(D_{E}^{k}\right)^{-\beta} C_{I}} \cdot \mathbf{g}_{E}^{k},
$$

where $\alpha_{0}$ is a constant dependent on the wireless propagation environment. In (1), $D_{I}$ and each $D_{E}^{k}$ represent the distance from the $\mathrm{BS}$ to the ID and $k$-th $\mathrm{EH}$ receiver respectively, and $\beta$ is the pathloss coefficient. $C_{I}$ and $C_{E}^{k}$ denote the shadowing effect. Each entry in $\mathbf{g}_{I}$ and $\mathbf{g}_{E}^{k}$ follows the standard complex Gaussian distribution, which forms the Rayleigh component of the channel.

For the conventional case where a fully-digital beamforming scheme is applied, we denote $\mathbf{W}=\left[\mathbf{w}_{I}, \mathbf{w}_{E}^{1}, \ldots, \mathbf{w}_{E}^{K}\right]$ as the fully-digital beamforming matrix. For the considered scenario of one ID and $K \mathrm{EH}$ receivers, since $\mathrm{EH}$ receivers do not need to decode the symbols, therefore energy beamforming is indeed not necessary and the optimal beamforming scheme is to employ the information beamforming only, which leads to $\mathbf{W}=\left[\mathbf{w}_{I}, \mathbf{0}, \ldots, \mathbf{0}\right]$. With this observation, the received signalto-noise ratio (SNR) $\gamma_{I}$ for the ID and the harvested energy $E_{k}$ for the $k$-th $\mathrm{EH}$ receiver can be expressed as

$$
\gamma_{I}=\frac{\left\|\mathbf{h}_{I} \mathbf{w}_{I}\right\|^{2}}{\sigma^{2}}, E_{k}=\eta\left(\left\|\mathbf{h}_{E}^{k} \mathbf{w}_{I}\right\|^{2}+\sigma^{2}\right),
$$

where $\sigma^{2}$ is the power of the additive Gaussian noise, and $\eta$ represents the efficiency of converting received radio signals into electrical energy. For simplicity in this paper we have assumed that each $\mathrm{EH}$ receiver has an identical energy transfer efficiency $\eta$ and harvested energy target $E_{0}$. Based on the above, we can formulate the transmit power minimization problem as

$$
\begin{array}{ll}
\mathcal{P}_{1}: & \min _{\mathbf{w}_{I}} p \\
\text { s.t. } & p \geq\left\|\mathbf{w}_{I}\right\|^{2} \\
& \gamma_{I} \geq \gamma_{0} \\
& E_{k} \geq E_{0}, \forall k \in \mathcal{K}
\end{array}
$$

By denoting $\mathbf{W}_{I}=\mathbf{w}_{I} \mathbf{w}_{I}^{H}, \mathbf{H}_{I}=\mathbf{h}_{I}^{H} \mathbf{h}_{I}$ and $\mathbf{H}_{E}^{k}=$ $\left(\mathbf{h}_{E}^{k}\right)^{H} \mathbf{h}_{E}^{k}, \mathcal{P}_{1}$ can be transformed into a semi-definite programming (SDP), given by

$$
\begin{array}{ll}
\mathcal{P}_{2}: & \min _{\mathbf{W}_{I}} \operatorname{tr}\left\{\mathbf{W}_{I}\right\} \\
\text { s.t. } & \operatorname{tr}\left\{\mathbf{H}_{I} \mathbf{W}_{I}\right\} \geq \gamma_{0} \sigma^{2} \\
& \operatorname{tr}\left\{\mathbf{H}_{E}^{k} \mathbf{W}_{I}\right\}+\sigma^{2} \geq \frac{E_{0}}{\eta}, \forall k \in \mathcal{K} \\
& \mathbf{W}_{I} \succeq 0, \operatorname{rank}\left\{\mathbf{W}_{I}\right\}=1
\end{array}
$$

By dropping the rank-1 constraint for $\mathbf{W}_{I}$, the relaxed $\mathcal{P}_{2}$ becomes convex and can be efficiently solved by convex optimization tools. Then, the beamforming vector $\mathbf{w}_{I}$ can be obtained via the eigenvalue decomposition of $\mathbf{W}_{I}$.

\section{Proposed Hybrid ANALOG-Digital SWIPT}

To reduce the total power consumption at the $\mathrm{BS}$ and achieve energy-efficient transmission, we propose a hybrid analog-digital beamforming scheme for SWIPT, where fullyconnected and partially-connected structures are considered. In this case, the beamforming matrix can be obtained as

$$
\mathbf{F}=\mathbf{F}_{R F} \mathbf{F}_{B B}
$$

where $\mathbf{F}_{B B} \in \mathcal{C}^{(K+1) \times(K+1)}$ denotes the low-dimensional digital beamformer, which can be decomposed as

$$
\mathbf{F}_{B B}=\left[\mathbf{f}_{I}, \mathbf{f}_{E}^{1}, \ldots, \mathbf{f}_{E}^{K}\right] .
$$

In (5), $\mathbf{F}_{R F} \in \mathcal{C}^{N_{t} \times(K+1)}$ is the analog beamformer implemented with phase shifters. When a fully-connected analog beamformer is considered [14], each entry of $\mathbf{F}_{R F}$ is of constant modulus and can be expressed as

$$
\left[\mathbf{F}_{R F}\right]_{m, n}=e^{j \varphi_{m, n}}
$$

For partially-connected structures [22], $\mathbf{F}_{R F}$ becomes a blockdiagonal matrix and $\mathbf{F}_{R F}=\operatorname{diag}\left(\mathbf{f}_{1}^{p}, \ldots, \mathbf{f}_{K+1}^{p}\right)$, with each entry of $\mathbf{f}_{k}^{p}$ given by

$$
\left[\mathbf{f}_{k}^{p}\right]_{m}=e^{j \varphi_{m, k}}
$$

Since it has been shown in Section II that the optimal fullydigital beamforming scheme is to employ the information beamformer only, it is then trivially obtained that for the hybrid beamforming scheme, the optimal low-dimensional digital beamforming scheme is still to employ the information beamformer only, i.e.

$$
\mathbf{F}_{B B}=\left[\mathbf{f}_{I}, \mathbf{0}, \ldots, \mathbf{0}\right]
$$

Then, the received SNR of the ID and harvested energy of each $\mathrm{EH}$ receiver for hybrid structures can be expressed as

$$
\gamma_{I}=\frac{\left\|\mathbf{h}_{I} \mathbf{F}_{R F} \mathbf{f}_{I}\right\|^{2}}{\sigma^{2}}, E_{k}=\eta\left(\left\|\mathbf{h}_{E}^{k} \mathbf{F}_{R F} \mathbf{f}_{I}\right\|^{2}+\sigma^{2}\right),
$$


and the power minimization problem with hybrid beamforming can be formulated as

$$
\begin{array}{ll}
\mathcal{P}_{3}: & \min _{\mathbf{F}_{R F}, \mathbf{f}_{I}}\left\|\mathbf{F}_{R F} \mathbf{f}_{I}\right\|^{2} \\
\text { s.t. } & \frac{\left\|\mathbf{h}_{I} \mathbf{F}_{R F} \mathbf{f}_{I}\right\|^{2}}{\sigma^{2}} \geq \gamma_{0} \\
& \eta\left(\left\|\mathbf{h}_{E}^{k} \mathbf{F}_{R F} \mathbf{f}_{I}\right\|^{2}+\sigma^{2}\right) \geq E_{0}, \forall k \in \mathcal{K} \\
& \mathbf{F}_{R F} \in \mathcal{F}
\end{array}
$$

where $\mathcal{F}$ is the set of matrices with each entry satisfying the constant modulus. The optimization $\mathcal{P}_{3}$ is difficult to solve due to the non-convex constant modulus constraint for the analog beamformer $\mathbf{F}_{R F}$. Nevertheless, we note that this problem can be efficiently transformed into an SDP to obtain $\mathbf{f}_{I}$ when $\mathbf{F}_{R F}$ is fixed, which motivates us to propose an iterative design where we alternately optimize $\mathbf{F}_{R F}$ and $\mathbf{f}_{I}$. To be specific, for the design of the analog beamformer $\mathbf{F}_{R F}$, we propose to minimize the difference between the optimal fullydigital beamformer and the hybrid beamformer [14], and the optimization problem can be formulated as

$$
\begin{array}{ll}
\mathcal{P}_{4}: & \min _{\mathbf{F}_{R F}}\left\|\mathbf{W}-\mathbf{F}_{R F} \mathbf{F}_{B B}\right\|^{2} \\
\text { s.t. } & \mathbf{F}_{R F} \in \mathcal{F}
\end{array}
$$

By denoting $\mathbf{F}_{R F}=\left[\left(\mathbf{f}_{R F}^{1}\right)^{T}, \ldots,\left(\mathbf{f}_{R F}^{N_{t}}\right)^{T}\right]^{T}$, the objective function of $\mathcal{P}_{4}$ can be further obtained as

$$
\begin{aligned}
& \left\|\mathbf{W}-\mathbf{F}_{R F} \mathbf{F}_{B B}\right\|^{2} \\
& =\left\|\left[\mathbf{w}_{I}, \mathbf{0}, \ldots, \mathbf{0}\right]-\mathbf{F}_{R F}\left[\mathbf{f}_{I}, \mathbf{0}, \ldots, \mathbf{0}\right]\right\|^{2} \\
& =\left\|\mathbf{w}_{I}-\mathbf{F}_{R F} \mathbf{f}_{I}\right\|^{2} \\
& =\sum_{i=1}^{N_{t}}\left\|\left[\mathbf{w}_{I}\right]_{i}-\mathbf{f}_{R F}^{i} \mathbf{f}_{I}\right\|^{2} \\
& =\sum_{i=1}^{N_{t}}\left\|\left[\mathbf{w}_{I}\right]_{i}-\sum_{m=1}^{K+1}\left[\mathbf{f}_{R F}^{i}\right]_{m}\left[\mathbf{f}_{I}\right]_{m}\right\|^{2},
\end{aligned}
$$

where we have decomposed $\mathbf{w}_{I}$ and $\mathbf{F}_{R F}$ by row. It is then observed in (13) that the objective function of $\mathcal{P}_{4}$ has been decomposed into $N_{t}$ independent sub-functions, and therefore $\mathcal{P}_{4}$ is equivalent to minimizing each of the $N_{t}$ sub-problems, which is formulated as

$$
\begin{array}{ll}
\mathcal{P}_{5}^{i}: & \min _{\mathbf{f}_{R F}^{i}}\left\|\left[\mathbf{w}_{I}\right]_{i}-\sum_{m=1}^{K+1}\left[\mathbf{f}_{R F}^{i}\right]_{m}\left[\mathbf{f}_{I}\right]_{m}\right\| \\
\text { s.t. } & \mathbf{f}_{R F}^{i} \in \mathcal{G}
\end{array}
$$

where $\mathcal{G}$ denotes the set of row vectors with each entry satisfying the constant modulus constraint.

\section{A. Optimal Solution for Analog Beamformer via Geometrical Interpretation}

As each entry in $\mathbf{f}_{R F}^{i}$ is of constant modulus, therefore the multiplication of each $\left[\mathbf{f}_{R F}^{i}\right]_{m}$ to the corresponding $\left[\mathbf{f}_{I}\right]_{m}$ is equivalent to an angle rotation of $\left[\mathbf{f}_{I}\right]_{m}$. Since $\left[\mathbf{w}_{I}\right]_{i}$ is a scalar, we can therefore obtain the optimal analog beamformer $\mathbf{f}_{R F}^{i}$ via a geometrical interpretation, as shown in Fig. 1.

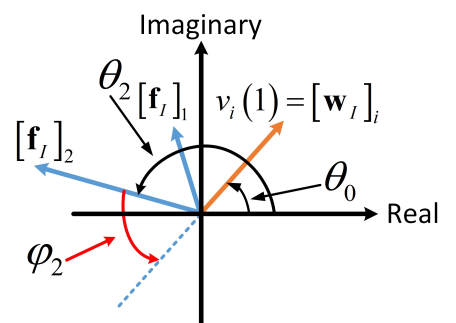

(a) Step 1: Rotate $\left[\mathbf{f}_{I}\right]_{2}$ to the opposite side of $\left[\mathbf{w}_{I}\right]_{i}$

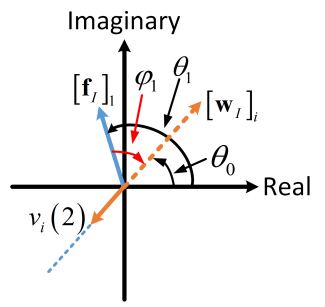

(b) Step 2: Rotate $\left[\mathbf{f}_{I}\right]_{1}$ to the same side of $\left[\mathbf{w}_{I}\right]_{i}$
Fig. 1: Geometrical interpretation of $\mathcal{P}_{5}^{i}$ and the solution

In Fig. 1, the dashed brown line denotes the fully-digital beamformer $\left[\mathbf{w}_{I}\right]_{i}$ with the phase of $\theta_{0}$, and the blue lines denote each $\left[\mathbf{f}_{I}\right]_{m}$ with phase $\theta_{m}$, where we assume $\theta_{0}, \theta_{m} \in$ $[0,2 \pi)$. It is then obvious that the optimal solution of $\mathbf{f}_{R F}^{i}$ is to rotate each $\mathbf{f}_{I}(m)$ such that the resulting $\mathbf{f}_{R F}^{i}(m) \mathbf{f}_{I}(m)$ is collinear to $\mathbf{w}_{I}(i) . \varphi_{m}$ then denotes the phase to be rotated for each $\left[\mathbf{f}_{I}\right]_{m}$ to achieve collinearity. To guarantee that the resulting objective function of $\mathcal{P}_{5}^{i}$ is minimized, we should firstly sort the elements in $\mathbf{f}_{I}$ in the descending order of amplitude, and the elements with a larger amplitude should be rotated first. An algorithm based on successive phase rotation is then proposed to efficiently solve $\mathcal{P}_{5}^{i}$. To be specific, the phase of each $\mathbf{f}_{R F}^{i}(m)$ to achieve collinearity can be obtained based on Fig. 1 as

$$
\varphi_{m}=\theta_{0}-\theta_{m}, \text { or } \varphi_{m}=\theta_{0}+\pi-\theta_{m},
$$

which is dependent on whether $\left[\mathbf{f}_{I}\right]_{m}$ is rotated to the same direction of $\left[\mathbf{w}_{I}\right]_{i}$ or the opposite direction. We further define the difference between the optimal fully-digital beamformer and the sum of previously rotated $m$ components of $\mathbf{f}_{I}$ as $v_{i}(m)$, given by

$$
v_{i}(m)=\left[\mathbf{w}_{I}\right]_{i}-\sum_{j=1}^{m-1}\left[\mathbf{f}_{R F}^{i}\right]_{j}\left[\mathbf{f}_{I}\right]_{j},
$$

which is a function of $m$ and denoted as the solid brown line in Fig. 1. In Fig. 1 (a), $\left[\mathbf{f}_{I}\right]_{2}$ should be rotated first since it has a larger amplitude and $\varphi_{2}=\theta_{0}+\pi-\theta_{2}$, which leads to Fig. 1 (b). Since the phase of $v_{i}(2)$ in Fig. 1 (b) is the opposite of $\left[\mathbf{w}_{I}\right]_{i}$, then $\left[\mathbf{f}_{I}\right]_{1}$ should be rotated to the same direction of $\left[\mathbf{w}_{I}\right]_{i}$ and $\varphi_{1}=\theta_{0}-\theta_{1}$. Indeed, based on the definition of $v_{i}(m)$, each $\left[\mathbf{f}_{I}\right]_{m}$ should be rotated to the opposite of $v_{i}(m)$ to further reduce the difference.

The above algorithm is summarized in Algorithm 1 below, where $\hat{\mathbf{a}}=\operatorname{sort}(\mathbf{a}, \mathrm{d})$ denotes the function that sorts each entry of $\mathbf{a}$ in a descending order of amplitude, and $\hat{\mathbf{a}}$ is the re-ordered vector. The function $x=$ find $\{a=\mathbf{b}\}$ means $[\mathbf{b}]_{x}=a$ and $\arg \{a\}$ is the phase of $a$. With $\mathbf{F}_{R F}$ obtained, we can efficiently solve $\mathcal{P}_{3}$ to obtain the low-dimensional digital beamformer by following a similar step from $\mathcal{P}_{1}$ to $\mathcal{P}_{2}$, and the details are omitted for brevity.

Noting that the above design of the analog beamformer requires the knowledge of $\mathbf{f}_{I}$, we therefore propose an iter- 


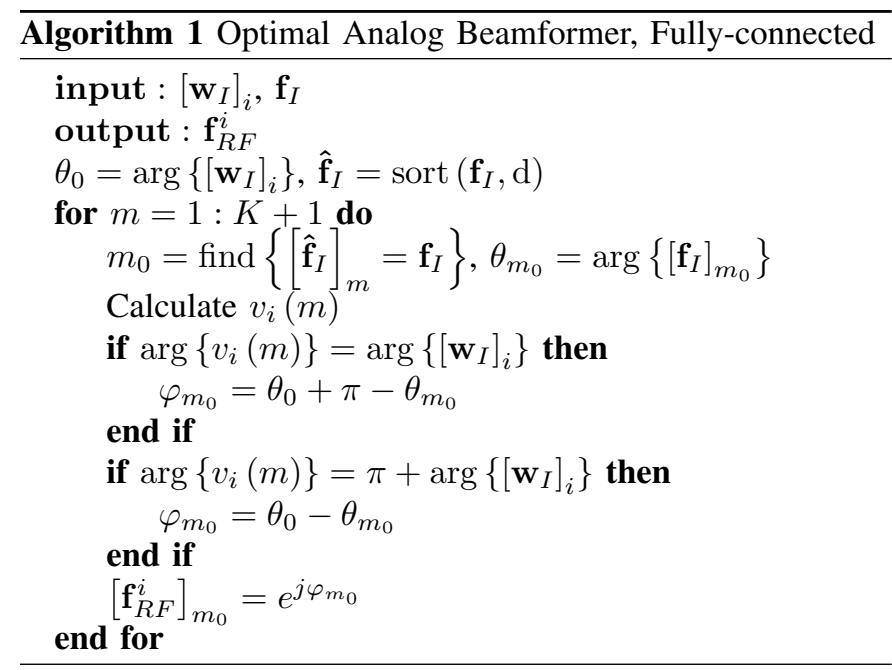

ative design where we alternately update $\mathbf{F}_{R F}$ and $\mathbf{f}_{I}$ until convergence or a maximum number of iterations is reached, shown below in Algorithm 2, where $\mathbf{f}_{I}^{0}$ is the initial lowdimensional digital beamformer, and $N_{\max }$ is the maximum iteration number. $\Delta$ is introduced to represent the convergence accuracy and $\Delta_{t h}$ denotes the accuracy threshold.

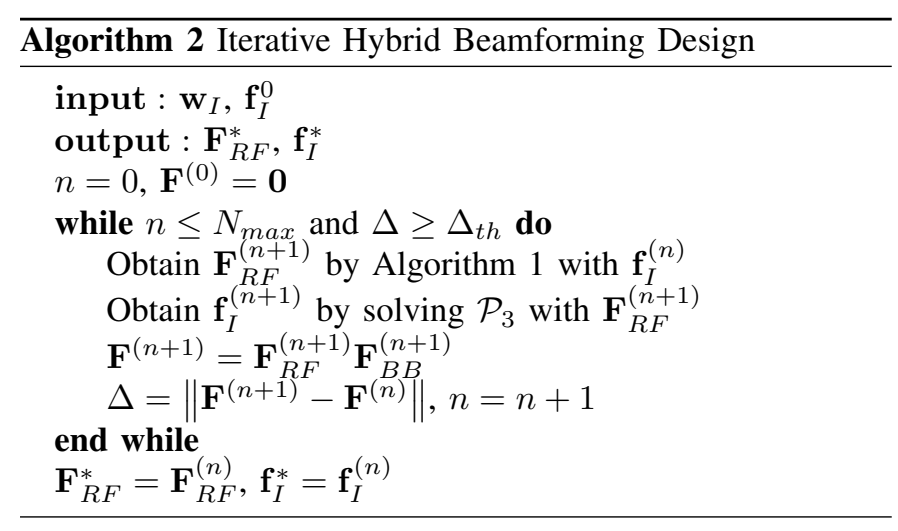

\section{B. Extension to Partially-Connected Structures}

With partially-connected structures, we note that each $\mathbf{f}_{R F}^{i}$ only has one non-zero element, and therefore we only need to rotate this entry to the opposite of $\left[\mathbf{w}_{I}\right]_{i}$, which simplifies the analog beamforming design. We then summarize the algorithm for partially-connected structures in Algorithm 3, where $\lceil x\rceil$ denotes the minimum integer that is not smaller than $x$.

\section{Power Consumption Model at the BS}

To evaluate the power savings by the hybrid beamforming structures, in this section we consider the total power consumption at the BS, which is modelled as [25]

$$
\begin{aligned}
P_{B S}^{\text {Digital }}= & N_{t}\left(N_{t}+1\right) P_{P A}+\left(N_{t}\right)^{2} P_{P S}+P_{B B} \\
& +N_{t}\left(P_{R F C}+P_{D A C}\right), \\
P_{B S}^{F u l l y}= & N_{t}\left(N_{R F}^{t}+1\right) P_{P A}+N_{t} N_{R F}^{t} P_{P S}+P_{B B} \\
+ & N_{R F}^{t}\left(P_{R F C}+P_{D A C}\right),
\end{aligned}
$$

$$
\begin{aligned}
P_{B S}^{\text {Partially }}= & N_{t} P_{P A}+N_{t} P_{P S}+P_{B B} \\
& +N_{R F}^{t}\left(P_{R F C}+P_{D A C}\right),
\end{aligned}
$$

where $P_{B S}^{\text {Digital }}, P_{B S}^{\text {Fully }}$ and $P_{B S}^{\text {Partially }}$ denote the total power consumption at the BS for fully-digital, fully-connected hybrid, and partially-connected hybrid structures, respectively. $P_{P A}=\left(1 / \eta_{0}\right) P_{T X}$ denotes the power consumed at the power amplifier to generate the transmit power $P_{T X}$, with $\eta_{0}$ being the power amplifier efficiency. $P_{P S}, P_{R F C}, P_{D A C}$ and $P_{B B}$ denote the power consumption for phase shifters, RF chains, digital-to-analog converters (DAC) and baseband processing, respectively.

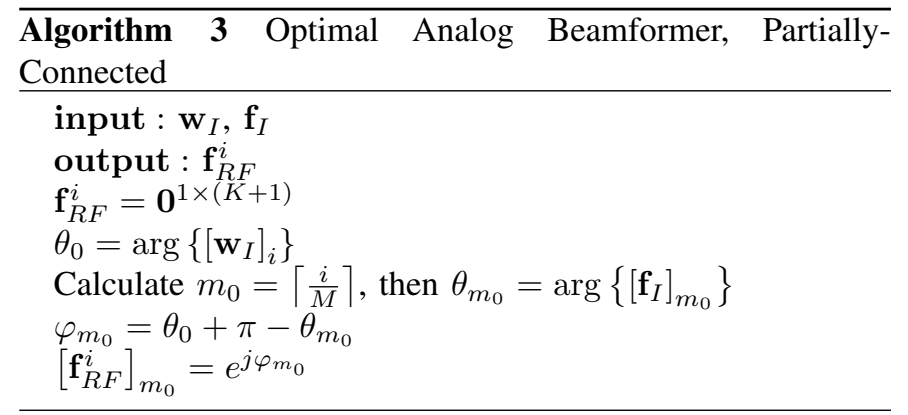

\section{Numerical Results}

We present numerical results based on Monte Carlo simulations in this section. For the channel modelling, following [24] we assume $\alpha_{0}=1, \beta=1, C_{I}=C_{E}^{k}=1, \forall k, D_{I}=10 \mathrm{~m}$, $D_{E}^{k}=5 \mathrm{~m}, \forall k$, and the noise power is assumed $\sigma^{2}=0.1 \mathrm{~mW}$. The energy transfer efficiency is $\eta=0.5$ and the power amplifier efficiency is $\eta_{0}=0.2$. The power consumption for each hardware component is $P_{P S}=30 \mathrm{~mW}, P_{R F C}=40 \mathrm{~mW}$, $P_{D A C}=200 \mathrm{~mW}$, and $P_{B B}=5 \mathrm{~mW}$ [25]. We compare the proposed scheme (denoted as 'Hybrid Iterative') with the fully-digital scheme ('Fully-Digital') and the hybrid scheme by phase conjugation [19] ('Hybrid PC').

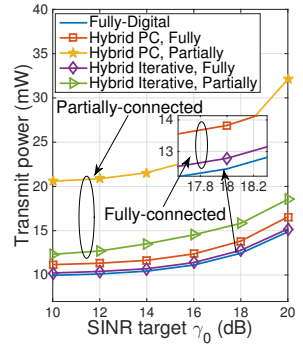

(a) Transmit power $P_{T X}$ $(\mathrm{mW})$

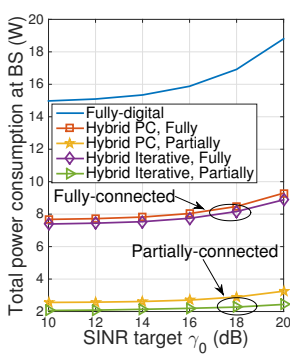

(b) Total power consumption at BS $P_{B S}(\mathrm{~W})$
Fig. 2: Transmit power and total power at BS required, $N_{t}=$ 12 , one ID, $K=3 \mathrm{EH}$ receivers, $E_{0}=5 \mathrm{~mW}, N_{\max }=6$

Fig. 2 presents the required transmit power $P_{T X}$ and the total power consumption $P_{B S}$ at the BS with respect to the SINR target of the ID and $E_{0}=5 \mathrm{~mW}$. Throughout the simulations, the proposed iterative scheme is usually convergent within 5 iterations. It is observed that the proposed iterative scheme with fully-connected structures can achieve a very close performance to the fully-digital case regarding the transmit power 
$P_{T X}$ due to the optimal analog beamforming design. It is also observed that the performance gains over phase conjugation are more significant for the partially-connected structures. Thanks to the reduced number of RF chains, hybrid structures are more promising regarding the total power consumption at the BS, as shown in Fig. 2 (b), especially for the partiallyconnected structures. A similar result can be observed in Fig. 3 where the power is shown with the increasing energy target of the EH receivers and $\gamma_{0}=14 \mathrm{~dB}$. While not shown due to limited space, the performance gains of the proposed iterative scheme over the low-complexity hybrid scheme by phase conjugation are more significant when the number of RF chains is smaller than $K+1$.

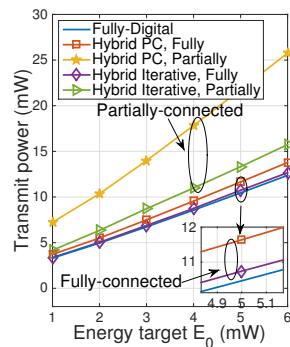

(a) Transmit power $P_{T X}$ $(\mathrm{mW})$

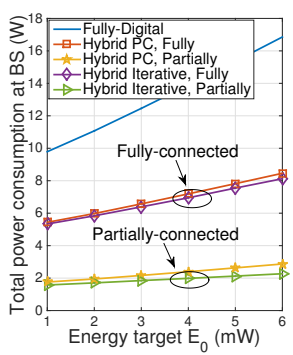

(b) Total power consumption at BS $P_{B S}(\mathrm{~W})$
Fig. 3: Transmit power and total power at BS required, $N_{t}=$ 12 , one ID, $K=3 \mathrm{EH}$ receivers, $\gamma_{0}=14 \mathrm{~dB}, N_{\max }=6$

\section{CONCLUSION}

In this paper, the energy-efficient SWIPT for MIMO systems with limited RF chains is studied. An iterative hybrid analog-digital beamforming scheme is proposed by minimizing the difference between the fully-digital beamformer and the hybrid beamformer, where the optimal analog beamformer structure is obtained by a geometrical representation. Numerical results show that the hybrid scheme achieves a near-optimal performance while consumes much less power compared to fully-digital SWIPT, and is therefore promising for the future energy-efficient transmission.

\section{ACKNOWLEDGMENT}

This work was supported by the Royal Academy of Engineering, U.K., the Engineering and Physical Sciences Research Council (EPSRC) project EP/M014150/1, and the China Scholarship Council (CSC).

\section{REFERENCES}

[1] P. Grover and A. Sahai, "Shannon Meets Tesla: Wireless Information and Power Transfer," in 2010 IEEE International Symposium on Information Theory, Austin, TX, 2010, pp. 2363-2367.

[2] R. Zhang and C. K. Ho, "MIMO Broadcasting for Simultaneously Wireless Information and Power Transfer," IEEE Trans. Wireless Commun., vol. 12, no. 5, pp. 1989-2001, May 2013.

[3] Z. Ding, C. Zhong, D. W. K. Ng, M. Peng, H. A. Suraweera, R. Schober, and H. V. Poor, "Application of Smart Antenna Technologies in Simultaneous Wireless Information and Power Transfer," IEEE Commun. Mag., vol. 53, no. 4, pp. 86-93, Apr. 2015.

[4] T. L. Thanh, M. D. Renzo, and J. P. Coon, "MIMO Cellular Networks with Simultaneous Wireless Information and Power Transfer," in 2016 IEEE 17th International Workshop on Signal Processing Advances in Wireless Communications (SPAWC), Edinburgh, 2016, pp. 1-5.
[5] M. Sheng, L. Wang, X. Wang, Y. Zhang, C. Xu, and J. Li, "Energy Efficient Beamforming in MISO Heterogeneous Cellular Networks with Wireless Information and Power Transfer," IEEE J. Sel. Areas Commun., vol. 34, no. 4, pp. 954-968, Apr. 2016.

[6] H. Son and B. Clerckx, "Joint Beamforming Design for Multi-User Wireless Information and Power Transfer," IEEE Trans. Wireless Commun., vol. 13, no. 11, pp. 6397-6409, Aug. 2014.

[7] W. Wu and B. Wang, "Robust Downlink Beamforming Design for Multiuser MISO Communication Systems with SWIPT," in 2015 IEEE International Conference on Communications (ICC), London, 2015, pp. 4751-4756.

[8] J. Liao, M. R. A. Khandaker, and K.-K. Wong, "Robust Power-Splitting SWIPT Beamforming for Broadcast Channels," IEEE Commun. Lett., vol. 20, no. 1, pp. 181-184, Jan. 2016.

[9] S. Timotheou, G. Zheng, C. Masouros, and I. Krikidis, "Exploiting Constructive Interference for Simultaneous Wireless Information and Power Transfer in Multiuser Downlink Systems," IEEE J. Sel. Areas Commun., vol. 34, no. 5, pp. 1772-1784, May 2016.

[10] Z. Zong, H. Feng, F. R. Yu, N. Zhao, T. Yang, and B. Hu, "Optimal Transceiver Design for SWIPT in K-User MIMO Interference Channels," IEEE Trans. Wireless Commun., vol. 15, no. 1, pp. 430-445, Jan. 2016.

[11] S. Lee, L. Liu, and R. Zhang, "Collaborative Wireless Energy and Information Transfer in Interference Channels," IEEE Trans. Wireless Commun., vol. 14, no. 1, pp. 545-557, Sept. 2015.

[12] X. Chen, D. W. K. Ng, and H.-H. Chen, "Secrecy Wireless Information and Power Transfer: Challenges and Opportunities," IEEE Wireless Commun., vol. 23, no. 2, pp. 54-61, May 2016.

[13] Q. Shi, W. Xu, J. Wu, E. Song, and Y. Wang, "Secure Beamforming for MIMO Broadcasting with Wireless Information and Power Transfer," IEEE Trans. Wireless Commun., vol. 14, no. 5, pp. 2841-2853, Jan. 2015.

[14] O. E. Ayach, S. Rajagopal, S. Abu-Surra, Z. Pi, and R. W. Heath, "Spatially Sparse Precoding in Millimeter Wave MIMO Systems," IEEE Trans. Wireless Commun., vol. 13, no. 3, pp. 1499-1513, Jan. 2014.

[15] P. V. Amadori and C. Masouros, "Low RF-Complexity Millimeter-Wave Beamspace-MIMO Systems by Beam Selection," IEEE Trans. Commun., vol. 63, no. 6, pp. 2212-2223, June 2015.

[16] A. Garcia-Rodriguez, C. Masouros, and P. Rulikowski, "Reduced Switching Connectivity for Power-Efficient large Scale Antenna Selection," IEEE Trans. Commun., DOI: 10.1109/TCOMM.2017.2669030 2017.

[17] A. Li, C. Masouros, and C. B. Papadias, "MIMO Transmission for Single-fed ESPAR with Quantized Loads," IEEE Trans. Commun., DOI: 10.1109/TCOMM.2017.26922242017.

[18] A. Li and C. Masouros, "Exploiting Constructive Mutual Coupling in P2P MIMO by Analog-Digital Phase Alignment," IEEE Trans. Wireless Commun., vol. 16, no. 3, pp. 1948-1962, Mar. 2017.

[19] L. Liang, W. Xu, and X. Dong, "Low-Complexity Hybrid Precoding in Massive Multiuser MIMO Systems," IEEE Wireless Commun. Lett., vol. 3, no. 6, pp. 653-656, Oct. 2014.

[20] V. Venkateswaran, F. Pivit, and L. Guan, "Hybrid RF and Digital Beamformer for Cellular Networks: Algorithms, Microwave Architectures, and Measurements," IEEE Trans. Microw. Theory and Techniques, vol. 64, no. 7, pp. 2226-2243, July 2016.

[21] A. Garcia-Rodriguez, V. Venkateswaran, P. Rulikowski, and C. Masouros, "Hybrid Analog-Digital Precoding Revisited under Realistic RF Modeling," IEEE Wireless Commun. Lett., vol. 5, no. 5, pp. 528-531, Oct. 2016.

[22] S. Han, C.-I. I, Z. Xu, and C. Rowell, "Large-Scale Antenna Systems with Hybrid Analog and Digital Beamforming for Millimeter Wave 5G,' IEEE Commun. Mag., vol. 53, no. 1, pp. 186-194, Jan. 2015.

[23] A. Li and C. Masouros, "Hybrid Analog-Digital Millimeter-Wave MUMIMO Transmission with Virtual Path Selection," IEEE Commun. Lett., vol. 21, no. 2, pp. 438-441, Feb. 2017.

[24] K. Yang, Q. Yu, S. Leng, B. Fan, and F. Wu, "Data and Energy Integrated Communication Networks for Wireless Big Data,' IEEE Access, vol. 4, pp. 713-723, Feb. 2016.

[25] R. Mendez-Rial, C. Rusu, N. Gonzalez-Prelcic, A. Alkhateeb, and R. W. Heath, "Hybrid MIMO Architectures for Millimeter Wave Communications: Phase Shifters or Switches?" IEEE Access, vol. 4, pp. 247-267, Jan. 2016 